\title{
Adaptação transcultural multicêntrica da sexta versão da Escala de Gravidade de Dependência (ASI6) para o Brasil
}

\author{
Multi-center cross-cultural adaptation of the Addiction Severity Index, Sixth Edition \\ (ASI6) for Brazil
}

\author{
Felix Kessler ${ }^{1}$, John Cacciola ${ }^{2}$, Sibele Faller ${ }^{1}$, Maria Lucia Souza-Formigoni ${ }^{3}$, Marcelo Cruz ${ }^{4}$, \\ Sílvia Brasiliano ${ }^{5}$, Flavio Pechansky ${ }^{1}$
}

${ }^{1}$ Centro de Pesquisa de Droga e Álcool, Universidade Federal do Rio Grande do Sul (UFRGS), Porto Alegre, RS. ${ }^{2}$ Departamento de Psiquiatria, University of Pennsylvania, Filadélfia, EUA. ${ }^{3}$ Departamento de Psicobiologia, Universidade Federal de São Paulo (UNIFESP), São Paulo, SP. ${ }^{4}$ Instituto de Psiquiatria, Universidade Federal do Rio de Janeiro (UFRJ), Rio de Janeiro, RJ. ${ }^{5}$ Instituto de Psiquiatria, Escola de Medicina, Universidade de São Paulo (USP), São Paulo, SP.

Financiamento: Este estudo foi apoiado pela Secretaria Nacional Antidrogas (SENAD), GPPG-HCPA nº 05-460.

Projeto aprovado pelo Comitê de Ética.

Prezados Editores:

O primeiro passo na avaliação de usuários de álcool e drogas é a obtenção de uma meticulosa e fiel avaliação diagnóstica biopsicossocial, promovendo o adequado desenvolvimento de um plano terapêutico. Embora tal conceito seja claro para profissionais brasileiros que trabalham no campo da dependência química, existe uma escassez de instrumentos de pesquisa e clínica com bom perfil psicométrico para a avaliação desses indivíduos especialmente escalas que abordem a vasta gama de problemas encontrados em usuários de álcool e drogas ${ }^{1}$.

Nesse contexto, a sexta versão do Addiction Severity Index (ASI6) ou Escala de Gravidade de Dependência foi escolhida para ser adaptada e validada no Brasil em um estudo multicêntrico. O ASI já foi traduzido para mais de 20 idiomas $^{2}$, demonstrando completa evidência de sua confiabilidade e validade ${ }^{3-5}$. Trata-se de uma entrevista semi-estruturada, planejada para obter informação sobre o perfil do abuso de substâncias e aspectos da vida relacionados com o uso de álcool e drogas nas seguintes áreas: médica, emprego/sustento, aspectos legais, sociofamiliar e psiquiátrica. Os entrevistadores, geralmente, levam de 45 a 90 minutos para concluir a aplicação do instrumento.

A última versão do instrumento (ASI6) foi desenvolvida para aperfeiçoar o conteúdo sobre problemas relacionados ao abuso de substâncias e abordar as principais limitações que estiveram presentes na sua versão anterior (ASI5) ${ }^{2}$.

Estudos estão sendo conduzidos nos EUA para validar a sexta versão do ASI, e os autores da escala original participaram ativamente, durante 2 anos, do desenvolvimento e adaptação do instrumento brasileiro, por meio de reuniões em ambos os países e correio eletrônico. O método de tradução e adaptação consistiu nas etapas mostradas na Figura 1.

Sendo o ASI um complexo questionário que exige treinamento específico, foi desenvolvido, em paralelo com o processo de adaptação do instrumento, um vídeo de treinamento e o manual do ASI6, onde foram fornecidas orientações sobre todos os itens, além de descrições e explicações detalhadas. A primeira versão brasileira do ASI6, com manual e vídeo, está disponível nos sites do Observatório Brasileiro de Informação sobre Drogas (OBID, www.obid.senad.gov.br) e do Centro de Pesquisas de Álcool e Drogas (CPAD, www.cpad.org.br). O download pode ser feito gratuitamente.

Este é o primeiro estudo conduzido na América Latina com o objetivo de adaptar e validar o ASI6. O trabalho envolveu esforços de pesquisadores de várias regiões do Brasil (Sul, Sudeste e Nordeste), originando também uma parceria para outros estudos nessa importante área da saúde pública. Com a adaptação do 


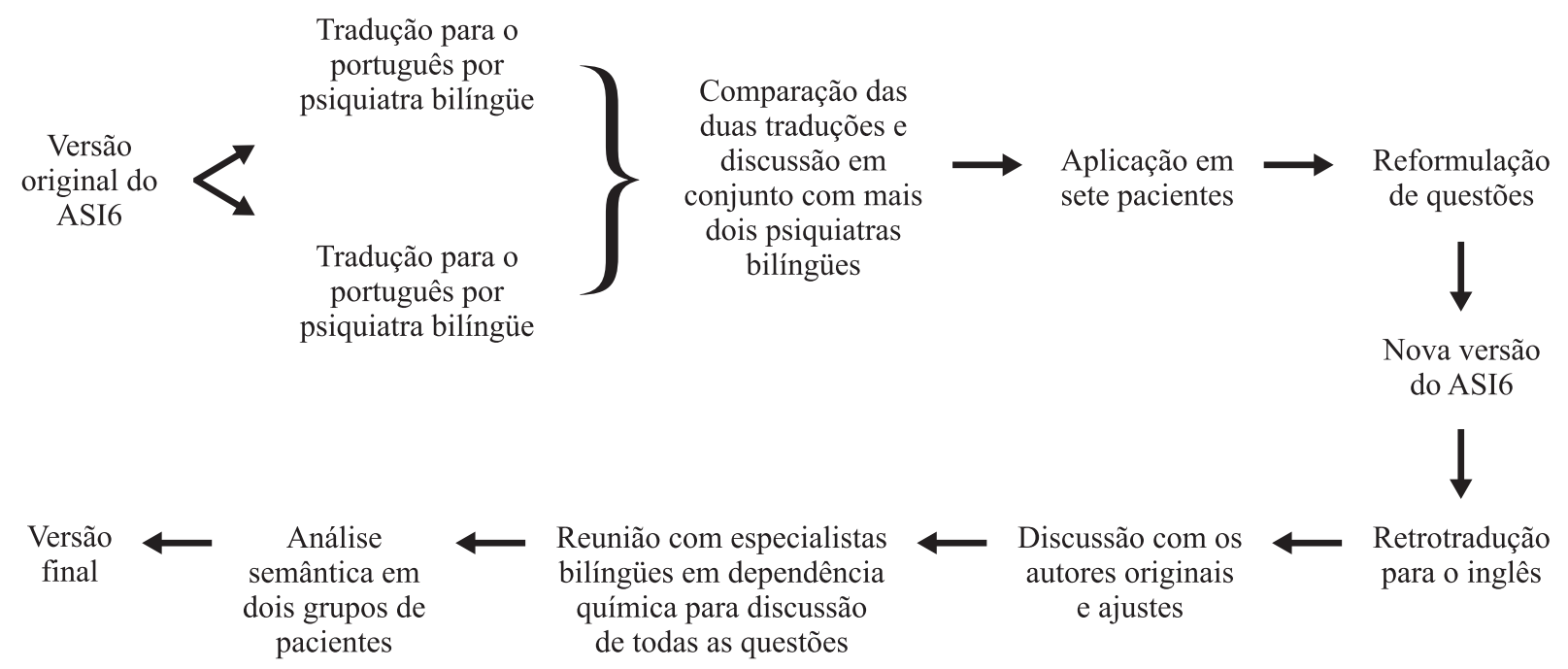

Todos os psiquiatras envolvidos são também especialistas em álcool e drogas.

Figura 1 - Apresentação das etapas metodológicas de tradução e adaptação transcultural da sexta versão da Escala de Gravidade de Dependência (ASI6) para o Brasil

ASI6 será possível treinar profissionais da saúde para obter melhor visualização dos problemas dos usuários de drogas, com fins de planejamento terapêutico, bem como para o seguimento de cada caso. Além disso, ela abre caminho para o desenvolvimento de outras versões do instrumento no Brasil, tais como a ASI "Lite", a ASI "follow-up" e uma versão informatizada. Uma iniciativa importante para testar o ASI6 no Brasil está em curso, de modo a validar o instrumento em um estudo multicêntrico no qual as propriedades psicométricas do instrumento (em amostras de usuários de álcool e/ou drogas de ambulatórios e internações de quatro estados do Brasil) serão investigadas.

\section{Referências}

1. Formigoni MLOS, Castel S. Escalas de avaliação de dependência de drogas: aspectos gerais. Rev Bras Psiquiatr. 1999;26(1):5-31.

2. Thomas McLellan A, Cacciola JC, Alterman AI, Rikoon SH, Carise D. The addiction severity index at 25: origins, contributions and transitions. Am J Addict. 2006;15(2):113-24.

3. Senoo E, Ogai Y, Haraguchi A, Kondo A, Ishibashi Y, Umeno M, et al. Reliability and validity of the Japanese version of the Addiction Severity Index (ASI-J). Nihon Arukoru Yakubutsu Igakkai Zasshi. 2006;41(4):368-79.

4. Scheurich A, Muller MJ, Wetzel H, Anghelescu I, Klawe C, Ruppe A, et al. Reliability and validity of the German version of the European Addiction Severity Index (EuropASI). J Stud Alcohol. 2000;61(6):916-9.

5. Gerevich J, Bácskai E, Kó J, Rózsa S. Reliability and validity of the Hungarian version of the European Addiction Severity Index. Psychopathology. 2005;38(6):301-9. 